\title{
Towards two-Ioop QCD corrections to ZZ and WW production at LHC
}

\author{
Thomas Gehrmann, Lorenzo Tancredi* and Erich Weihs \\ Institut für Theoretische Physik, Universität Zürich, Wintherturerstrasse 190, \\ CH-8057 Zürich, Switzerland \\ E-mail: thomas.gehrmann@uzh.ch, tancredi@physik.uzh.ch, \\ erich.weihs@physik.uzh.ch
}

We report on the progress towards the computation of the two-loop QCD corrections to ZZ and WW production at the LHC. The latter involve a new class of Feynman integrals: two-loop fourpoint functions with two off-shell external legs. We describe their reduction to a small set of master integrals and their computation by the method of differential equations.

11th International Symposium on Radiative Corrections (Applications of Quantum Field Theory to Phenomenology) (RADCOR 2013),

22-27 September 2013

Lumley Castle Hotel, Durham, UK

\footnotetext{
* Speaker.
} 


\section{Introduction and method}

Vector boson pair production $(\gamma \gamma, Z \gamma / W \gamma, Z Z, W W, W Z)$ is a key process in studying the dynamics of the electroweak theory at the LHC. It enters as background not only for Higgs production, but also for many other new-physics searches as it offers a large number of observables which allow precise tests of the electroweak symmetry-breaking and in general of the non-abelian gauge structure of the group $S U(2) \times U(1)$. With large production rates to be expected from the future data taking at the LHC, vector boson pair production processes will become electroweak precision observables. The high experimental precision must be matched by a comparably high accuracy of the theoretical predictions, typically requiring next-to-leading order (NLO) electroweak and next-to-next-to-leading order (NNLO) QCD corrections. At present the massless NNLO QCD corrections are known only for $\gamma \gamma$ and $Z \gamma$ production [1,2].

A full NNLO computation requires three different ingredients: the two-loop double-virtual corrections to the partonic $2 \rightarrow 2$ process, the one-loop real-virtual corrections to the $2 \rightarrow 3$ process for the production of the vector boson pair plus an additional parton, and the tree-level corrections to the $2 \rightarrow 4$ process involving two extra partons.

In the case of vector boson pair production, the $2 \rightarrow 3$ and $2 \rightarrow 4$ ingredients have already been known in the literature for some time [3-10]. On the other hand, the two-loop parton-level matrix elements are known only for $\gamma \gamma[11]$ and $Z \gamma / W \gamma$ production $[12,13]$. Finally, in the case of $W W$ production the two-loop virtual corrections are known in the high-energy approximation [14].

The analytic computation of the two-loop matrix elements for massive vector boson pair production is still an outstanding task and it proceeds as follows [15]. Consider the $2 \rightarrow 2$ process:

$$
q\left(p_{1}\right)+\bar{q}\left(p_{2}\right) \rightarrow V\left(q_{1}\right)+V\left(q_{2}\right)
$$

where $V$ are the massive vector bosons and:

$$
p_{1}^{2}=p_{2}^{2}=0, \quad q_{1}^{2}=q_{2}^{2}=m^{2} .
$$

Defining the usual Mandelstam variables in the Minkowski region:

$$
s=\left(p_{1}+p_{2}\right)^{2}=m^{2} \frac{(1+x)^{2}}{x}, \quad t=\left(p_{2}-q_{1}\right)^{2}=-m^{2} y, \quad u=\left(p_{2}-q_{1}\right)^{2}=-m^{2} z
$$

then the physical region for vector boson pair production is given by (see fig.(1)):

$$
0<x<1, \quad x<z<\frac{1}{x} .
$$

Starting from the Feynman diagrammatic representation of the two-loop amplitude, we identify the two-loop scalar integrals that contribute to the process. The latter can be organised in two planar topologies, that we named Topo A and Topo B, and a third topology, named Topo C, which contains all non-planar integrals. We choose the propagators of the three topologies as listed in Table 1.

Employing integration-by-parts (IBPs), Lorentz and symmetry identities [16, 17], together with the Laporta algorithm [18] as implemented in Reduze2 [19,20], we expressed all integrals as combination of a small number of Master Integrals (MIs). 


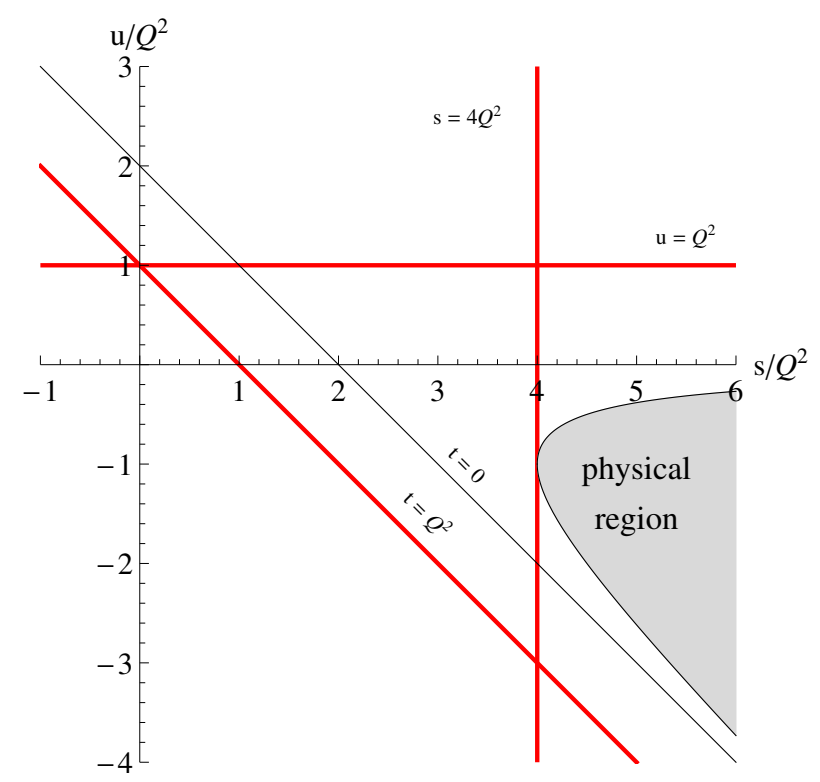

Figure 1: Dalitz plot for the $2 \rightarrow 2$ scattering kinematics.

$\begin{array}{lll}\text { Topo A } & \text { Topo B } & \text { Topo C } \\ k^{2} & k^{2} & k^{2} \\ l^{2} & l^{2} & l^{2} \\ (k-l)^{2} & (k-l)^{2} & (k-l)^{2} \\ \left(k-p_{1}\right)^{2} & \left(k-p_{1}\right)^{2} & \left(k-p_{1}\right)^{2} \\ \left(l-p_{1}\right)^{2} & \left(l-p_{1}\right)^{2} & \left(l-p_{1}\right)^{2} \\ \left(k-p_{1}-p_{2}\right)^{2} & \left(k-p_{1}+q_{1}\right)^{2} & \left(k-p_{1}-p_{2}\right)^{2} \\ \left(l-p_{1}-p_{2}\right)^{2} & \left(l-p_{1}+q_{1}\right)^{2} & \left(k-l-q_{1}\right)^{2} \\ \left(k-p_{1}-p_{2}+q_{1}\right)^{2} & \left(k-p_{1}-p_{2}+q_{1}\right)^{2} & \left(l-p_{1}-p_{2}+q_{1}\right)^{2} \\ \left(l-p_{1}-p_{2}+q_{1}\right)^{2} & \left(l-p_{1}-p_{2}+q_{1}\right)^{2} & \left(k-l-p_{1}-p_{2}\right)^{2}\end{array}$

Table 1: Propagators in the three different auxiliary topologies used to represent all two-loop 4-point integrals with two massless and two massive legs with the same mass.

The computation of the latter is performed with the differential equation method [21-24]. Differential equations for the integrals under consideration are derived at the basis of the integrands. The master integrals are then determined by solving these differential equations, matched to appropriate boundary conditions (that usually correspond to integrals in special points or with fewer scales). By direct inspection of the differential equations it becomes clear that all MIs can be expressed in terms of Generalised Harmonic PolyLogarithms (GHPLs) [25-29]. In order to determine the boundary conditions and to perform necessary transformations on the differential equations, we made extensive use of the Symbol and Co-product formalism [30-32]. 


\section{The planar master integrals}

As reported in [15], we computed all planar two-loop master integrals contributing to $\mathrm{ZZ}$ and WW production in massless QCD. The first topology, Topo A, contains 26 genuine two-loop MIs, out of which 13 were new double-boxes. The second topology, Topo B, contains instead 13 genuine two-loop MIs and 9 new double-boxes. The double-boxes are:
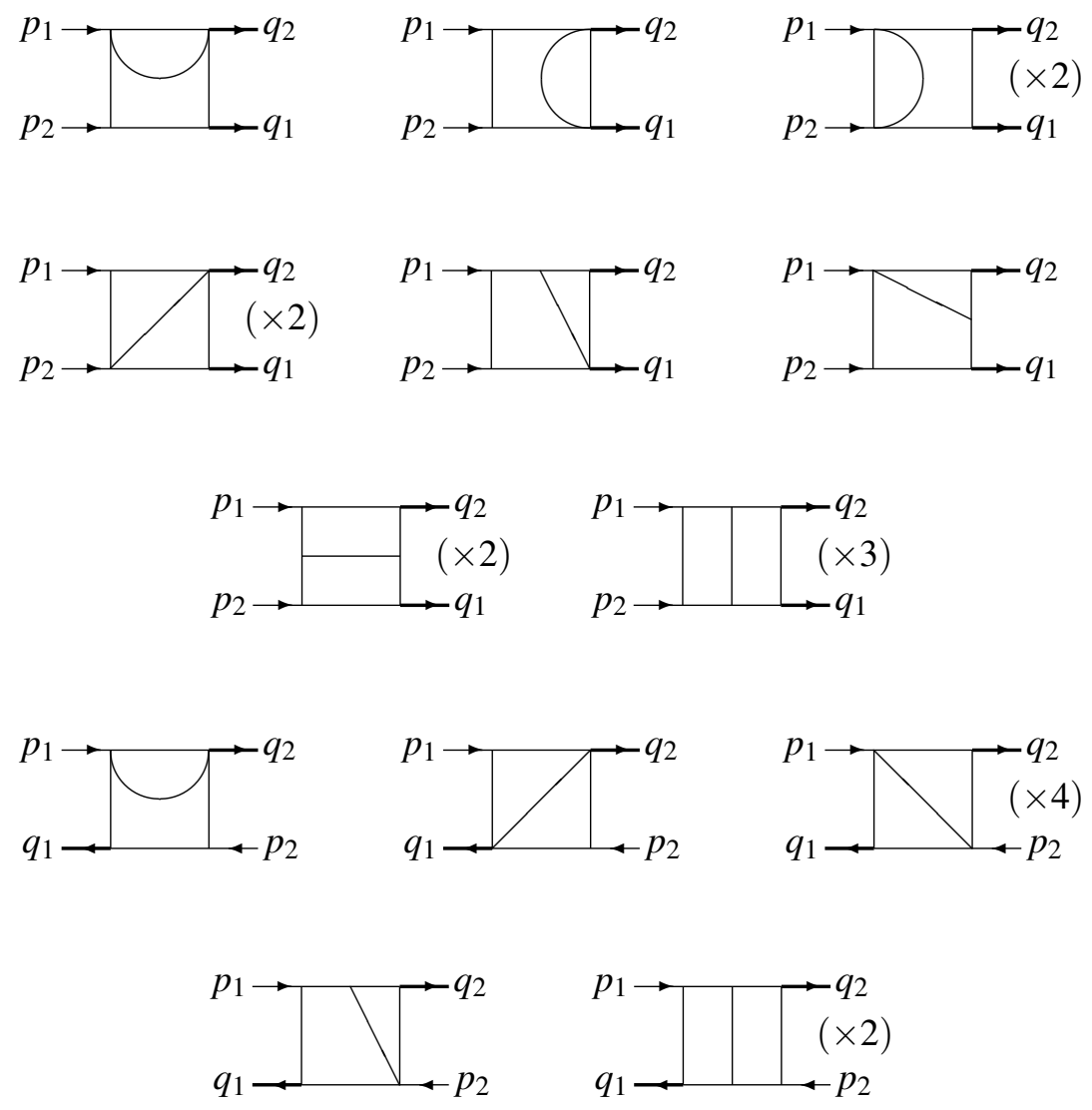

The numbers in brackets indicate that the corresponding sector is reduced to more than one MI.

Employing the variables defined above (1.1), we find that all MIs in both topologies can be expressed in compact form as function of GHPLs with the following alphabet:

$$
\begin{aligned}
G(\vec{f}(x) ; z) \quad \text { with } \quad f_{j}(x) & =\left\{1,0,-1, x, \frac{1}{x}, \frac{x}{1+x+x^{2}}, \frac{1+x+x^{2}}{x}, \frac{1+x^{2}}{x}\right\}, \\
G(\vec{a} ; x) \text { with } \quad a_{j} & =\left\{1,0,-1,+i,-i, \frac{1+i \sqrt{3}}{2}, \frac{1-i \sqrt{3}}{2}\right\} .
\end{aligned}
$$

Similar sets of GHPLs have been already encountered in the computation of the two-loop corrections to $t \bar{t}$ production [33-36]. The presence of non-linear indices in the GHPLs comes directly from the non-linear kinematical constraints in (1). 


\section{Status on the computation of the non-planar master integrals}

The computation of the non-planar MIs proceeds in principle in the very same way as outlined in the section above. Performing the reduction to MIs we find that this topology contains 16 genuine two-loop MIs, out of which 13 are new double-boxes that still need to be computed.

In deriving the differential equations for all integrals in this topology, it turns out to be easy to find a basis of MIs for which the differential equations decouple (at least in the limit for $d \rightarrow 4$ ) for all those sectors which contain more than one MI. With any choice of MIs that respects this basic requirement, the integration of the differential equations themselves, and so the determination of the functional form of the result, remains as straightforward as in the planar case. In particular, as already stated before, by direct inspection of the equations, it becomes clear that the very same set of GHPLs employed for representing the planar integrals (2.1), (2.2), is enough also for representing all non-planar integrals.

Nevertheless, the analytic structure of non-planar integrals is more involved, since, as it is well known, integrals belonging to non-planar topologies are expected to have cuts in all three Mandelstam variables $(s, t, u)$ at the same time. This feature makes in general more difficult the determination of the boundary conditions needed for fixing the exact solution of the differential equations.

In order to handle the complexity of this computation it might turn out to be useful to organize it following the strategy outlined in [37] (see also the proceedings of this conference). There are indications that a basis for the non-planar integrals, which respects the basic requirements in [37] can be found, and so that the system of differential equations can be put in the general form:

$$
\begin{aligned}
& \partial_{x} f(\varepsilon ; x, z)=\varepsilon A_{x}(x, z) f(\varepsilon ; x, z) \\
& \partial_{z} f(\varepsilon ; x, z)=\varepsilon A_{z}(x, z) f(\varepsilon ; x, z)
\end{aligned}
$$

where $\varepsilon=(4-d) / 2$ is the dimensional regularisation parameter, $f(\varepsilon ; x, z)$ is the vector of all master integrals in the topology, and $A_{x}(x, z), A_{z}(x, z)$ are matrices whose entries do not depend on $\varepsilon$. The computation of the non-planar MIs is currently ongoing.

\section{Conclusions}

The computation of the two-loop corrections to the production of a pair of massive vector bosons has been for a long time an outstanding task, principally due to the complexity of the functions needed to represent the result, namely the generalized harmonic polylogarithms. In the last years considerable progress has be made in the understanding of the algebraic and analytic properties of these functions, so that the task appears finally within reach. We presented new results for the planar master integrals required for the computation of the leading color contribution to $Z Z$ and $W W$ production at two loop and showed who the symbol and coproduct formalism can be successfully employed in order to perform this computation. The study of the remaining nonplanar topologies is still ongoing. 


\section{References}

[1] S. Catani, L. Cieri, D. de Florian, G. Ferrera, and M. Grazzini, Diphoton production at hadron colliders: a fully-differential QCD calculation at NNLO, Phys.Rev.Lett. 108 (2012) 072001, [arXiv:1110.2375].

[2] M. Grazzini, S. Kallweit, D. Rathlev, and A. Torre, Z $\gamma$ production at hadron colliders in NNLO QCD, arXiv:1309.7000.

[3] V. Del Duca, F. Maltoni, Z. Nagy, and Z. Trocsanyi, QCD radiative corrections to prompt diphoton production in association with a jet at hadron colliders, JHEP 0304 (2003) 059, [hep-ph/0303012].

[4] T. Gehrmann, N. Greiner, and G. Heinrich, Photon isolation effects at NLO in gamma gamma + jet final states in hadronic collisions, JHEP 1306 (2013) 058, [a rXiv: 1303.0824 ].

[5] S. Dittmaier, S. Kallweit, and P. Uwer, NLO QCD corrections to $W W+j e t$ production at hadron colliders, Phys.Rev.Lett. 100 (2008) 062003, [arXiv: 0710 . 1577].

[6] S. Dittmaier, S. Kallweit, and P. Uwer, NLO QCD corrections to $p p / p \bar{p} \rightarrow W W+j e t+X$ including leptonic W-boson decays, Nucl.Phys. B826 (2010) 18-70, [arXiv: 0908 .4124].

[7] T. Binoth, T. Gleisberg, S. Karg, N. Kauer, and G. Sanguinetti, NLO QCD corrections to ZZ+ jet production at hadron colliders, Phys.Lett. B683 (2010) 154-159, [arXiv: 0911.3181$].$

[8] F. Campanario, C. Englert, M. Spannowsky, and D. Zeppenfeld, NLO-QCD corrections to $W \gamma j$ production, Europhys.Lett. 88 (2009) 11001, [arXiv:0908.1638].

[9] F. Campanario, C. Englert, and M. Spannowsky, Precise predictions for (non-standard) W $\gamma+$ jet production, Phys.Rev. D83 (2011) 074009, [arXiv: 1010.1291$].$

[10] F. Campanario, C. Englert, S. Kallweit, M. Spannowsky, and D. Zeppenfeld, NLO QCD corrections to WZ+jet production with leptonic decays, JHEP 1007 (2010) 076, [arXiv: 1006.0390$].$

[11] Z. Bern, A. De Freitas, and L. J. Dixon, Two loop amplitudes for gluon fusion into two photons, JHEP 0109 (2001) 037, [hep-ph/ 0109078 ].

[12] T. Gehrmann and L. Tancredi, Two-loop QCD helicity amplitudes for $q \bar{q} \rightarrow W^{ \pm} \gamma$ and $q \bar{q} \rightarrow Z^{0} \gamma$, JHEP 1202 (2012) 004, [arXiv: 1112.1531].

[13] T. Gehrmann, L. Tancredi, and E. Weihs, Two-loop QCD helicity amplitudes for $g \mathrm{~g} \rightarrow Z \mathrm{~g}$ and $g g \rightarrow Z \gamma$, JHEP 1304 (2013) 101, [arXiv: 1302 .2630].

[14] G. Chachamis, M. Czakon, and D. Eiras, W Pair Production at the LHC. I. Two-loop Corrections in the High Energy Limit, JHEP 0812 (2008) 003, [arXiv: 0802 . 4 028].

[15] T. Gehrmann, L. Tancredi, and E. Weihs, Two-loop master integrals for $q \bar{q} \rightarrow V V$ : the planar topologies, JHEP 1308 (2013) 070, [arXiv: 1306.6344 ].

[16] F. Tkachov, A Theorem on Analytical Calculability of Four Loop Renormalization Group Functions, Phys.Lett. B100 (1981) 65-68.

[17] K. Chetyrkin and F. Tkachov, Integration by Parts: The Algorithm to Calculate beta Functions in 4 Loops, Nucl.Phys. B192 (1981) 159-204.

[18] S. Laporta, High precision calculation of multiloop Feynman integrals by difference equations, Int.J.Mod.Phys. A15 (2000) 5087-5159, [hep-ph/ 0102033 ]. 
[19] C. Studerus, Reduze-Feynman Integral Reduction in C++, Comput.Phys.Commun. 181 (2010) 1293-1300, [arXiv:0912.2546].

[20] A. von Manteuffel and C. Studerus, Reduze 2 - Distributed Feynman Integral Reduction, arXiv:1201.4330.

[21] A. Kotikov, Differential equations method: New technique for massive Feynman diagrams calculation, Phys.Lett. B254 (1991) 158-164.

[22] E. Remiddi, Differential equations for Feynman graph amplitudes, Nuovo Cim. A110 (1997) 1435-1452, [hep-th/9711188].

[23] M. Caffo, H. Czyz, S. Laporta, and E. Remiddi, The Master differential equations for the two loop sunrise selfmass amplitudes, Nuovo Cim. A111 (1998) 365-389, [hep-th/9805118].

[24] T. Gehrmann and E. Remiddi, Differential equations for two loop four point functions, Nucl.Phys. B580 (2000) 485-518, [hep-ph/9912329].

[25] E. Remiddi and J. Vermaseren, Harmonic polylogarithms, Int.J.Mod.Phys. A15 (2000) 725-754, [hep-ph/9905237].

[26] T. Gehrmann and E. Remiddi, Two loop master integrals for $\gamma^{*} \rightarrow 3$ jets: The Planar topologies, Nucl.Phys. B601 (2001) 248-286, [hep-ph/ 0008287 ].

[27] T. Gehrmann and E. Remiddi, Two loop master integrals for $\gamma^{*} \rightarrow 3$ jets: The Nonplanar topologies, Nucl.Phys. B601 (2001) 287-317, [hep-ph/ 0101124$].$

[28] A. Goncharov, Multiple polylogarithms and mixed Tate motives, math/ 0103059.

[29] J. Vollinga and S. Weinzierl, Numerical evaluation of multiple polylogarithms, Comput.Phys.Commun. 167 (2005) 177, [hep-ph/ 0410259 ].

[30] F. Brown, The Massless higher-loop two-point function, Commun.Math.Phys. 287 (2009) 925-958, [arXiv:0804.1660].

[31] C. Duhr, Hopf algebras, coproducts and symbols: an application to Higgs boson amplitudes, JHEP 1208 (2012) 043, [arXiv: 1203.0454 ].

[32] C. Anastasiou, C. Duhr, F. Dulat, and B. Mistlberger, Soft triple-real radiation for Higgs production at N3LO, JHEP 1307 (2013) 003, [arXiv: 1302 . 4379].

[33] R. Bonciani, A. Ferroglia, T. Gehrmann, D. Maitre, and C. Studerus, Two-Loop Fermionic Corrections to Heavy-Quark Pair Production: The Quark-Antiquark Channel, JHEP 0807 (2008) 129, [arXiv:0806.2301].

[34] R. Bonciani, A. Ferroglia, T. Gehrmann, and C. Studerus, Two-Loop Planar Corrections to Heavy-Quark Pair Production in the Quark-Antiquark Channel, JHEP 0908 (2009) 067, [arXiv:0906.3671].

[35] R. Bonciani, A. Ferroglia, T. Gehrmann, A. Manteuffel, and C. Studerus, Two-Loop Leading Color Corrections to Heavy-Quark Pair Production in the Gluon Fusion Channel, JHEP 1101 (2011) 102, [arXiv:1011.6661].

[36] A. von Manteuffel and C. Studerus, Massive planar and non-planar double box integrals for light $N_{f}$ contributions to $g g \rightarrow t \bar{t}$, JHEP 1310 (2013) 037, [arXiv:1306.3504].

[37] J. M. Henn, Multiloop integrals in dimensional regularization made simple, Phys.Rev.Lett. 110 (2013) 251601, [arXiv:1304.1806]. 